\title{
Long-term survival after successful out-of-hospital resuscitation
}

\author{
G.P. Kimman ${ }^{\text {a }}$, E.M.A. Ivens ${ }^{a}$, J.A.M. Hartman ${ }^{\text {, }}$,H.N. Hart ${ }^{\text {, }}$ M.L. Simoons*a \\ a'Thoraxcenter, Erasmus University and University Hospital, Rotterdam, The Netherlands \\ ${ }^{b}$ Municipal Health Service, Rotterdam, The Netherlands
}

Received 26 April 1994; revision received 12 August 1994; accepted 15 September 1994

\begin{abstract}
Between 1983 and 1989, 962 patients in Rotterdam were resuscitated outside hospital, of whom $240(25 \%)$ could be discharged alive. A follow-up study was performed to determine prognosis in these patients. Of the 240 survivors of out-of-hospital resuscitation $80 \%$ survived after 1 year and $61 \%$ after 5 years. During the first year, $9 \%$ suffered from myocardial (re)infarction and $13 \%$ underwent coronary bypass surgery or angioplasty. Within the first 3 years after resuscitation $60 \%$ of the patients were readmitted to hospital. Permanent or temporary neurological deficits were observed in 30 patients (14\%). Patients with a primary arrhythmia without myocardial infarction had a worse prognosis than patients with a cardiac arrest in the context of an infarct. Survival was better in patients in whom resuscitation was initiated by physicians or ambulance-nurses, than in patients resuscitated by lay-people. Multivariate analysis revealed that this difference could be explained by a larger proportion of patients with a primary arrhythmia in the latter group. Since long-term prognosis after out-of-hospital resuscitation is satisfactory, programmes for resuscitation courses should be stimulated. Such programmes should aim predominantly at relatives of patients with known heart disease, police officers and children.
\end{abstract}

Keywords: Resuscitation; Prognosis; Ambulance service; Bystander; Out-of-hospital cardiac arrest

\section{Introduction}

Every year many people are victims of out-ofhospital cardiac arrest, caused by ventricular tachy-arrhythmias, brady-arrhythmias or asystole [1,2]. Most patients will die unless cardiopulmonary resuscitation (CPR) is started promptly [3]. In the last decades help at the time of a cardiac

\footnotetext{
* Corresponding author, Erasmus University, Thoraxcenter, Room BD 434, P.O. Box 1738, 3000 DR Rotterdam, The Netherlands. Tel.: 3110463 3938; Fax: 31104635258
}

arrest has improved because of the development of defibrillators and proper training of ambulancenurses in cardiopulmonary resuscitation [4]. Still, the time interval between cardiac arrest and arrival of emergency medical service teams was often too long and too late to administer help. Therefore, programmes for resuscitation training were developed for lay-men [5-7].

In 1979 a programme was established to teach the inhabitants of Rotterdam the elementary technique of CPR. In the past 15 years more then 50000 people have been trained out of a popula- 
tion of about one million. In this area there are 315 general practitioners. During daytime 14 emergency medical service teams are available, and during the evening and night there are 8 and 4 , respectively. Each year about 4500 patients are transported by emergency medical service teams to a coronary care unit, mostly $(80 \%)$ upon request by general practitioners, who visit patients at home with possible cardiac symptoms or collapse and who decide whether or not an emergency medical service team is needed. Approximately $30 \%$ of all out-of-hospital resuscitations are started by lay-men.

In this study we investigated long-term survival after bystander CPR, the event-free period of patients discharged alive and neurological deficits persisting after the arrest. Finally we analyzed predictors of long-term survival and compared bystander CPR with resuscitation started by emergency paramedics and physicians.

\section{Methods}

The Municipal Health Service in Rotterdam registers all out-of-hospital resuscitations. In the period between 1983 and 1989, 962 patients were resuscitated out-of-hospital by either emergency paramedics, physicians or lay-men (Table 1). Most patients (768) were in cardiac arrest at the time of arrival of the emergency medical service teams and 194 developed arrest in the presence of ambulancenurses or during transport to the hospital. All patients were taken over by the emergency medical service teams for transport to a hospital. Of these patients another 142 died in the hospital and 240 patients were finally discharged alive.

Follow-up data were collected through the Municipal Health Service, Population Registries, the hospitals where the patients were admitted, and from the general practitioners. In particular, it was assessed whether or not a patient was still

Table 1

Characteristics of 933 patients resuscitated out-of-hospital in Rotterdam in the period 1983-1989 by emergency paramedics, physicians and lay-men

\begin{tabular}{|c|c|c|c|c|c|}
\hline & \multicolumn{2}{|c|}{ Nurses ambulance service } & \multirow[t]{2}{*}{ Physicians } & \multirow[t]{2}{*}{ Lay-men } & \multirow[t]{2}{*}{ Total } \\
\hline & $\begin{array}{l}\text { arrest pre- } \\
\text { arrival }\end{array}$ & $\begin{array}{l}\text { arrest after } \\
\text { arrival }\end{array}$ & & & \\
\hline Total resuscitations & 207 & 194 & 246 & 286 & 933 \\
\hline Patients admitted to hospital & $62(30)$ & $120(62)$ & $88(36)$ & $100(35)$ & $370(40)$ \\
\hline Patients discharged alive & $44(21)$ & $68(35)$ & $70(28)$ & $48(17)$ & $230(25)$ \\
\hline Females $^{b}$ & $9(20)$ & $24(35)$ & $14(20)$ & $4(8)$ & $51(22)$ \\
\hline Males $^{b}$ & $35(80)$ & $44(65)$ & $56(80)$ & $44(92)$ & $179(78)$ \\
\hline Age (mean) ${ }^{b}$ & 61 & 67 & 61 & 66 & 64 \\
\hline History of myocardial infarction ${ }^{b}$ & $11(28)$ & $25(41)$ & $13(21)$ & $13(30)$ & $62(30)$ \\
\hline Infarction at time of arrest ${ }^{b}$ & $30(77)$ & $52(85)$ & $45(73)$ & $23(53)$ & $150(73)$ \\
\hline \multicolumn{6}{|l|}{ Symptoms and medication at discharge $e^{c}$} \\
\hline $\begin{array}{l}\text { Heart failure: digoxine, diuretics and/or } \\
\text { ACE inhibitors }\end{array}$ & $20(51)$ & $26(43)$ & $27(42)$ & $29(67)$ & $102(50)$ \\
\hline $\begin{array}{l}\text { Angina pectoris: nitrates, beta blockers } \\
\text { and/or Calcium channel blockers }\end{array}$ & $30(77)$ & $46(75)$ & $51(82)$ & $27(63)$ & $154(75)$ \\
\hline Anti-arrhythmics & $7(18)$ & $4(7)$ & $16(26)$ & $9(21)$ & $36(18)$ \\
\hline
\end{tabular}

Between brackets - percentages of patients in that column, related to total resuscitations (a); patients discharged from hospital (b), respectively 44, 68, 70, 48, 230 patients; patients with complete discharge information (c), respectively $39,61,62,43,205$ patients in each column.

In 29 cases resuscitation was initiated by others including 10 patients discharged ( 6 females, 4 males) who were reanimated by other paramedics or nurses. 
alive at follow-up or the date and cause of death. Hospital discharge- and out-patient letters were collected to obtain the final diagnosis and data about re-admissions. The presence or absence of possible neurological deficits, angina or heart failure were noticed. The general practitioners were asked by questionnaire to describe the present situation of the patients by focussing on symptoms of heart failure, angina, and neurological disturbances, as well as possible re-infarction, coronary bypass surgery, PTCA or other re-admissions. The same questionnaire was presented by telephone to those patients of whom the general practitioners were unknown.

\section{Results}

Of the 962 patients who were resuscitated outof-hospital in the period between 1983 and 1989, 623 patients had ventricular fibrillation and 339 patients had an asystole: 118 and 76 patients, respectively, developed ventricular fibrillation or asystole in the presence of emergency medical service teams. Most patients $(60 \%)$ died outside hospital and 382 patients reached the hospital alive. Finally $240(25 \%)$ were discharged alive: 183 men and 57 women, with a mean age of 63 years (range 15-88 years). Most men were between the ages of 50 and 79 years with a peak between 60 and 69 years. In the small group of women no specific age pattern was recognizable. A total of 222 patients $(93 \%)$ had ventricular fibrillation at time of arrival of the emergency health service teams or developed ventricular fibrillation in the presence of the ambulance-nurses. The other 18 patients $(7 \%)$ had other arrhythmias, including ventricular tachycardia, asystole and extreme bradycardia. The initial success rate was highest for patients who developed cardiac arrest in the presence of ambulance nurses $(62 \%)$ (Table 1$)$. This group also had the highest percentage of patients discharged alive. In contrast, of those patients who had a cardiac arrest before arrival of the emergency health services or who were resuscitated by lay-men $21 \%$ and $17 \%$ were discharged alive.

Survival at 1, 3 and 5 years after discharge was, respectively 80,67 and $61 \%$ (Fig. 1). Patients with a primary arrhythmia had a significantly worse
SURVIVAL PATIENTS DISCHARGED ALIVE

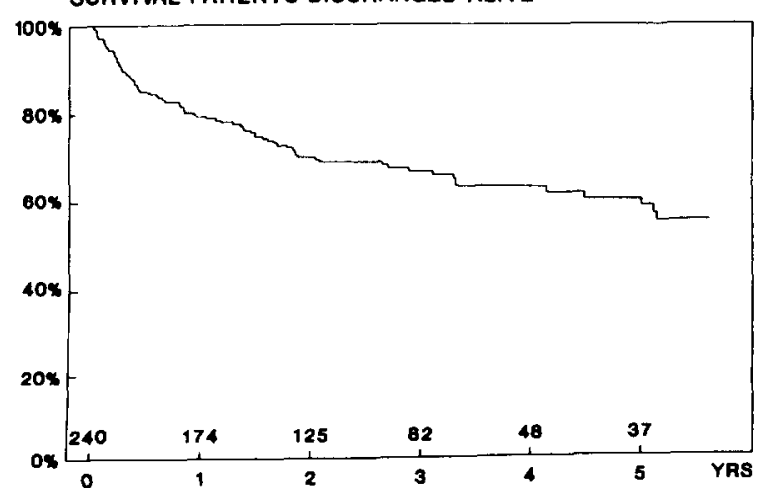

Fig. 1. Survival curve of 240 patients discharged alive after outof-hospital resuscitation in Rotterdam in the period 1983-1989. The number of patients alive at follow-up is indicated for each year.

prognosis than those with a myocardial infarction (Fig. 2, Mantel-Cox analysis: $P<0.01$ ). Within this first year after discharge 67 patients (32\%) were re-admitted to the hospital because of cardiac reasons, 18 patients $(9 \%)$ suffered from a myocardial (re)infarction, $17(9 \%)$ underwent bypass surgery and 8 patients (4\%) underwent PTCA.

At 1.5 years follow-up, $52 \%$ of all patients were alive without re-admissions because of cardiac

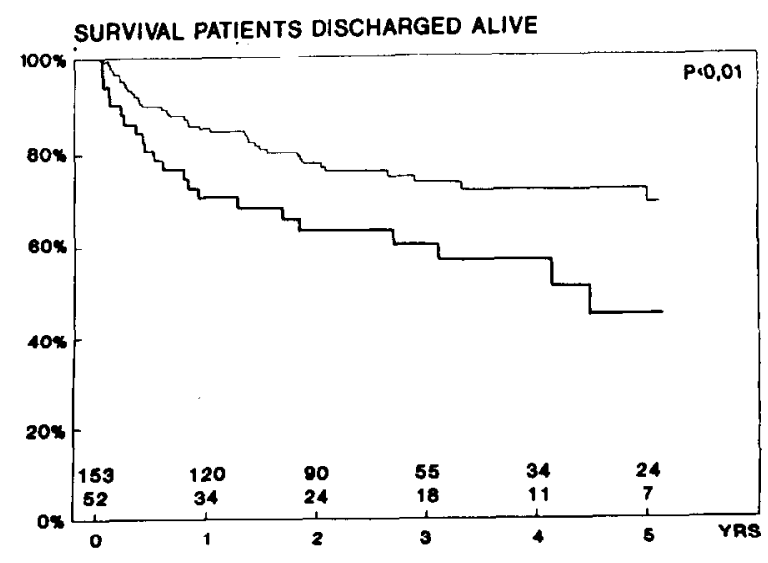

Fig. 2. Survival curves of 205 patients discharged after out-ofhospital resuscitation (see Fig. 1) separating patients with cardiac arrest as a result of myocardial infarction ( $n=52$, bottom curve) and patients with a primary cardiac arrest, without infarction ( $n=153$, top curve). Of 35 patients no diagnosis was available. 
Table 2

Course of neurological disturbances of 210 patients resuscitated in Rotterdam, in the period 1983-1989, after being discharged alive

\begin{tabular}{lllll}
\hline & $\begin{array}{l}\text { Impaired } \\
\text { memory }\end{array}$ & $\begin{array}{l}\text { Emotional } \\
\text { disturbances }\end{array}$ & $\begin{array}{l}\text { Physical } \\
\text { problems }\end{array}$ & Total \\
\hline $\begin{array}{l}\text { No. of patients } \\
\text { Present state*: }\end{array}$ & 22 & 13 & 8 & $30(14 \%)$ \\
Better & 9 & 3 & 4 & \\
Same & 7 & 4 & 3 & \\
Worse & 6 & 6 & 1 & \\
\hline
\end{tabular}

"Comparison with the situation after hospital discharge.

disease. At 5 years this figure was $35 \%$. Neurological findings after discharge were registered for 210 patients (Table 2). Only 30 of them (14\%) had neurological deficits - mostly impaired memory function.

Resuscitation was started by ambulance nurses in 112 patients $(47 \%)$ discharged alive, 44 of whom had a cardiac arrest before arrival of the ambulance while 68 patients developed cardiac arrest in the presence of the team. For 70 patients $(29 \%)$ resuscitation was started by General Practitioners, for 48 patients $(20 \%)$ by lay-men and for 10 patients $(4 \%)$ by paramedics, not connected to an emergency medical service team. There was no difference in the type of arrhythmias at the beginning of resuscitation between these groups. All $240 \mathrm{pa}$ tients were finally taken over by ambulance nurses and transported to the hospital. Long-term survival of patients initially resuscitated by physicians and patients who developed a cardiac arrest in the presence of the emergency team was similar. In contrast patients resuscitated by lay-men had a worse survival (Fig. 3). This difference in longterm survival can be explained in part by differences in patients characteristics (Table 1). In the group of patients resuscitated by lay-men myocardial infarction was less frequent and primary arrhythmias were more prominent than in the other groups $\left(\chi^{2}\right.$-test: $\left.p<0.05\right)$. In the former group there were more males and the percentage of patients treated for heart failure with digoxin, diuretics or ACE-inhibitors was higher, although these differences were not statistically significant.

A Cox multivariate analysis including the factors age, male gender, previous myocardial infarc- tion, diagnosis and those persons who started resuscitation showed that the differences in longterm survival were related to the diagnosis and not to the other factors. The lower long-term survival of patients resuscitated by lay-men can thus be explained by the fact that this group included more patients with primary arrhythmia.

\section{Discussion}

Outcome of out-of hospital resuscitation is mainly determined by the time-interval between the onset of cardiac arrest and the start of resuscitation and, furthermore, by the underlying heart disease, by the quality of resuscitation and the

SURVIVAL PATIENTS DISCHARGED ALIVE

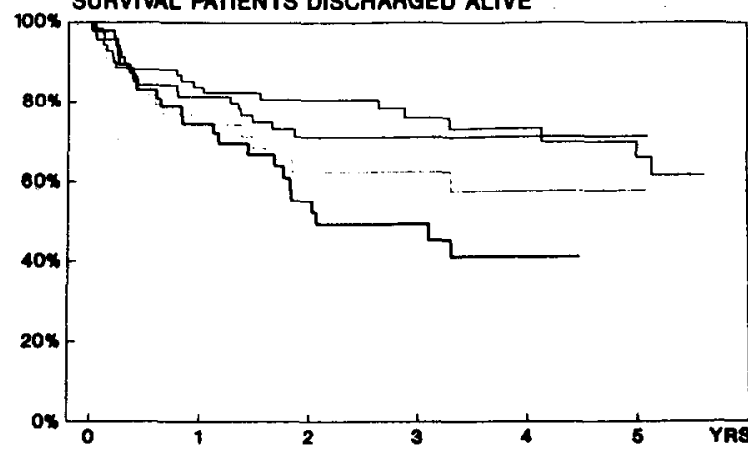

Fig. 3. Survival curves of 230 patients after out-of-hospital resuscitation: from top to bottom - patients who suffered from a cardiac arrest in the presence of emergency paramedics ( $n=68$ ), patients resuscitated by physicians $(n=70)$, patients who had a cardiac arrest before arrival of emergency paramedics $(n=44)$ and patients resuscitated by lay-men $(n=48)$. 
moment of defibrillation. Resuscitation is usually successful when cardiac arrest develops in the presence of emergency paramedics, nurses or physicians with appropriate medication and defibrillator immediately available. Resuscitation after a cardiac arrest in the presence of bystanders has a higher percentage of survival than resuscitation of patients who are found with cardiac arrest of uncertain duration.

In this study most patients who were resuscitated by lay-men or physicians, before arrival of the ambulance nurses, suffered from a cardiac arrest in their presence, although this was not registered. For witnessed cardiac arrests, the time from collapse to initiation of CPR was not registered either. Furthermore, no detailed information was available about the response time of an emergency medical service team at the time of a cardiac arrest, but probably this was about 5-10 min. Patients, who developed cardiac arrest in the presence of professionals (emergency nurses and physicians) had higher survival rates. Of all patients $40 \%$ reached the hospital alive and $25 \%$ were discharged alive. The primary success rate $(40 \%)$ is comparable with other studies $[1,3,11]$.

After successful out-of-hospital resuscitation patients have a favourable long-term survival: after 5 years $61 \%$ was still alive. These observations are similar to data from Seattle [8] where long-term survival of a comparable group of patients was $81 \%$ at 1 year and $49 \%$ at 4 years. In our series this was $80 \%$ and $64 \%$, respectively. The percentage of males was in both studies about the same, $76 \%$, while the patients in Seattle were on average 2 years older.

Most patients who suffered from cardiac arrest have extensive coronary disease, which explains the high percentage of subsequent cardiovascular morbidity. In the first year $9 \%$ of the patients had a (new) myocardial infarction and 13\% underwent bypass-surgery or PTCA.

Our high discharge rate can also be explained by the fact that most patients suffered from a myocardial infarction at the time of the cardiac arrest instead of a primary arrhythmia, which is accompanied by a far worse prognosis (maybe because of a higher recurrence rate of ventricular fibrillation due to a chronic process) [8]. Those pa- tients probably had prodromal symptoms prior to the cardiac arrest and therefore warned their general practitioners or the emergency medical service earlier by comparison with those who suffered from a cardiac arrest because of a primary arrhythmia.

After resuscitation part of the patients had neurological disturbances for a short or longer period. In spite of this, long-term neurological deficits were reported by physicians in only $14 \%$ of patients. It is difficult to compare this with other studies because of the different definitions used for neurological deficits [9-12].

It is remarkable that by univariate analysis patients resuscitated by lay-men seemed to have a worse long-term outcome after discharge than those resuscitated by physicians or emergency nurses. A lower initial success rate of resuscitation by lay-men might be caused by inadequate CPR technique compared with professionals. Other factors, such as patient selection, can also be responsible for this observation. In fact, patients resuscitated by lay-men had more often a cardiac arrest because of a primary arrhythmia. Furthermore these patients were older and had more often a history of previous myocardial infarction. After correction for these factors, by multivariate analysis, the long-term survival difference of patients resuscitated by lay-men or professionals was no longer apparent.

Since long-term prognosis after out-of-hospital resuscitation by lay-men is satisfactory, teaching programmes for resuscitation should be stimulated. At present $28 \%$ of all out-of-hospital resuscitations in Rotterdam are started by laymen. In most cities this is less than $10 \%$, while in Seattle a percentage of $60 \%$ has been reached after intensive campaigns [8]. Resuscitation courses should be aimed predominantly at relatives of patients with known heart disease because these have the highest risk for cardiac arrest. Police officers too should be trained in CPR, because they are often the first ones to be warned when someone gets unwell in public. Finally it seems advisable to teach CPR to children following secondary education. In Rotterdam such programmes have been started recently. People who followed such a resuscitation course in the past should be encouraged to 
follow regular retraining courses. Finally, systematic registration of out-of-hospital reanimation efforts may help to develop the most efficient programmes in different rural and city environments.

\section{References}

[1] Meyerburg RJ, Kessler KM, Zaman L, Conde CA, Castellanos A. Survivors of prehospital cardiac arrest. J Am Med Assoc 1982; 247: 1485-1490.

[2] de Luna AB, Coumel P, Leclerq JF. Amnbulatory sudden cardiac death: mechanisms of production of fatal arrhythmia on the basis of data from 157 cases. Am Heart J 1989; 117: 151-159.

[3] Ritter G, Wolfe RA, Goldstein S et al. The effect of bystander CPR on survival of out-of-hospital cardiac arrest victims. Am Heart J 1985; 110: 932-937.

[4] Kouwenhoven WB, Jude JR, Knickerbocker GG. Closed chest cardiac arrest resuscitation. J Am Med Assoc 1960; 173: 1064.

[5] Alvarez H, Cobb LA. Experience with CPR training of the general public. Washington DC: AHA/Red Cross Conference for Standards on CPR and ECG, May 16-18, 1973.
[6] Flax P, Larke T, Walser G, Kaye W, Uhley H. The mechanics of widespread training of cardiopulmonary resuscitation: a community project implemented by volunteers. Am Heart J 1976; 91: 123.

[7] Hart HN, Slooff R. Medical aspects of lay resuscitations in Rotterdam. Eur Heart $\mathrm{J}$ 1988; 9: 859-65.

[8] Eisenberg MS, Hallstrom A, Bergner L. Long-term survival after out-of hospital cardiac arrest. N Engl J Med 1982; 306: 1340-1343.

[9] Longstreth Jr. WT, Inui TS, Cobb LA, Copass MK. Neurological recovery after out-of-hospital cardiac arrest. Ann Intern Med 1983; 98 (part 1): 588-592.

[10] Earnest MP, Yarnell PR, Merrill BA, Knapp BA. Longterm survival and neurologic status after resuscitation from out-of-hospital cardiac arrest. Neurology 1980; 30: 1298-1302.

[11] Guzy PM, Pearce ML, Greenfield S. The survival of bystander cardiopulmonary resuscitation in a paramedic served metropolitan area. Am J Publ Health 1983; 73 : 766-769.

[12] Cobb LA, Baum, RS, Alvarez III HA, Schaffer WA. Resuscitation from out-of-hospital ventricular fibrillation: 4 years follow-up. Circulation 1985 (suppl III); 51, 52. 